\title{
Path Integral Formulation for Wave Effect in Multilens System
}

\author{
Kazuhiro Yamamoto \\ Department of Physical Science, Hiroshima University, Higashi-Hiroshima, Japan \\ Email: kazuhiro@hiroshima-u.ac.jp
}

How to cite this paper: Yamamoto, K. (2017) Path Integral Formulation for Wave Effect in Multilens System. International Journal of Astronomy and Astrophysics, 7, 221-229.

https://doi.org/10.4236/ijaa.2017.73018

Received: July 30, 2017

Accepted: September 19, 2017

Published: September 22, 2017

Copyright $\odot 2017$ by author and Scientific Research Publishing Inc. This work is licensed under the Creative Commons Attribution International License (CC BY 4.0).

http://creativecommons.org/licenses/by/4.0/

(c) (i) Open Access

\begin{abstract}
A formula to investigate the wave effect in a multi-lens system is presented on the basis of a path integral formalism by generalizing the work by Nakamura and Deguchi (1999). The wave effect of a system with two lenses is investigated in an analytic way as a simple application to demonstrate usefulness of the formula and variety of wave effect in multi-lens system.
\end{abstract}

\section{Keywords}

Cosmology, Gravitational Lensing, Wave Optics

\section{Introduction}

Recently several authors have investigated wave effect in gravitational lensing phenomenon, motivated by the possibility that the wave effect might be detected in future gravitational wave observatories [1]-[7]. The wave effect can be investigated by solving wave equation, in principle. However, it is difficult to solve the wave equation analytically for general configuration of lenses, excepting the simple case of a single Schwarzschild lens (e.g., [8] [9], see also [10] and references therein, cf. [3]). Indeed, investigation of the wave effect so far is restricted to special case of single lens model with the spherical symmetry, i.e., the Schwarzschild lens model and the singular isothermal sphere lens model (see [1]).

Nakamura and Deguchi developed an elegant formalism for gravitational lens using the path integral approach [6]. The primary purpose of the present paper is to derive a useful formula to investigate the wave effect in general multi-lens system, by generalizing the formalism by Nakamura and Deguchi. We also apply it to a system with two Schwarzschild lenses to demonstrate usefulness of the formula. This paper is organized as follows: In Section 2, we present a 
generalized formula for multi-lens system. In Section 3, an application of the formula to a simple configuration with two lenses is considered. Section 4 is devoted to summary and conclusion. We use the convention $c=1$.

\section{Generalized Formulation}

We start by reviewing the path integral formalism for gravitational lens phenomenon [6]. We consider the Newtonian spacetime with the metric

$$
\mathrm{d} s^{2}=-(1+2 U(r, \theta, \varphi)) \mathrm{d} t^{2}+(1-2 U(r, \theta, \varphi))\left(\mathrm{d} r^{2}+r^{2} \mathrm{~d} \theta^{2}+r^{2} \sin ^{2} \theta \mathrm{d} \varphi^{2}\right),
$$

where $U(r, \theta, \varphi)$ is the Newtonian potential. Propagation of massless field $\phi$ is described by the wave equation

$$
\frac{\partial}{\partial x^{\mu}}\left(\sqrt{-g} g^{\mu \nu} \frac{\partial}{\partial x^{v}}\right) \phi(\boldsymbol{r}, t)=0,
$$

where $g$ is the determinant of the metric and $\boldsymbol{r}$ represents the spatial coordinates $(r, \theta, \varphi)$. We consider a monochromatic wave from a point source with the wave number $k$. We set

$$
\phi(\boldsymbol{r}, t)=\frac{A}{r} F(\boldsymbol{r}) \mathrm{e}^{-i k(t-r)},
$$

where $A$ is a constant, then Equation (2) yields

$$
\frac{\partial^{2} F}{\partial r^{2}}+2 i k \frac{\partial F}{\partial r}+\frac{1}{r^{2}} \frac{1}{\sin \theta} \frac{\partial}{\partial \theta}\left(\sin \theta \frac{\partial F}{\partial \theta}\right)+\frac{1}{r^{2}} \frac{1}{\sin ^{2} \theta} \frac{\partial^{2} F}{\partial \varphi^{2}}-4 k^{2} U(r, \theta, \varphi) F=0 .
$$

Assuming that the first term is negligible compared with the second term and $\theta \ll 1$, Equation (4) reduces to

$$
i \frac{\partial F}{\partial r}=-\frac{1}{2} k r^{2}\left[\frac{1}{\theta} \frac{\partial}{\partial \theta}\left(\theta \frac{\partial F}{\partial \theta}\right)+\frac{1}{\theta^{2}} \frac{\partial^{2} F}{\partial \varphi^{2}}\right]+2 k U(r, \theta, \varphi) F .
$$

Due to analogy of Equation (5) with the Schrödinger equation, using the path integral formulation of quantum mechanics, the solution can be written as follows,

$$
F(r, \theta, \varphi)=\int \mathcal{D} \Theta \exp \left[i k \int_{0}^{r} \mathrm{~d} r\left(\frac{1}{2} r^{2} \dot{\Theta}^{2}(r)-2 U(r, \Theta(r))\right)\right],
$$

where the dot denotes the differentiation with respect to $r$ and $\Theta$ is used to represent the variables $(\theta, \varphi)$, which are related by $\Theta=(\theta \cos \varphi, \theta \sin \varphi)$. The expression (6) means the sum of all possible path $\Theta(r)$, fixing the initial point (source's position) and the final point (observer's position).

Let us consider multi-lens system, including $n$ lenses as shown in Figure 1, in which the source is located at the origin of coordinate and an observer is located at $(r, \Theta)=\left(r_{N}, \Theta_{N}\right)$. The radius between the source and the observer is discretized by $N$ segments, and each discrete radius from the source is labeled by $r_{j}$ with $j$ from 1 to $N$. Separation neighboring two discrete radii is $\varepsilon$. We assume that the $n$ lenses are located at the radius $r=r_{l_{m}}$ with $m$ from 1 to $n$, and that the thin lens approximation is valid for the lenses. In this case the 


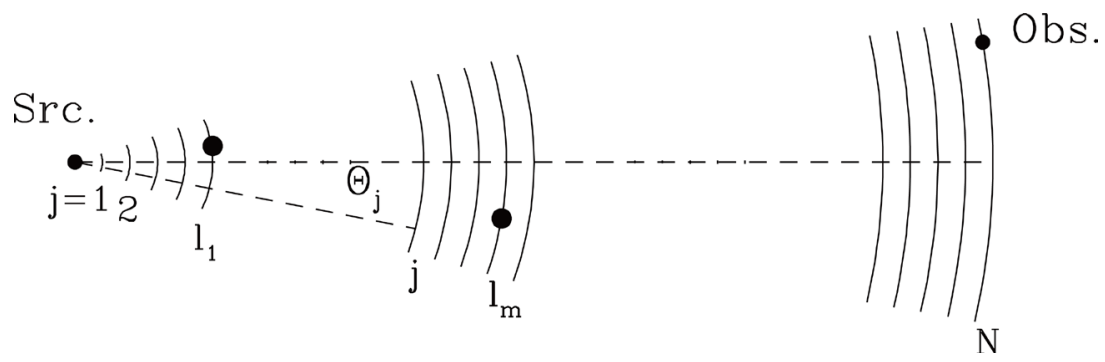

Figure 1. Configuration of multi-lens system and coordinates for path integral formula.

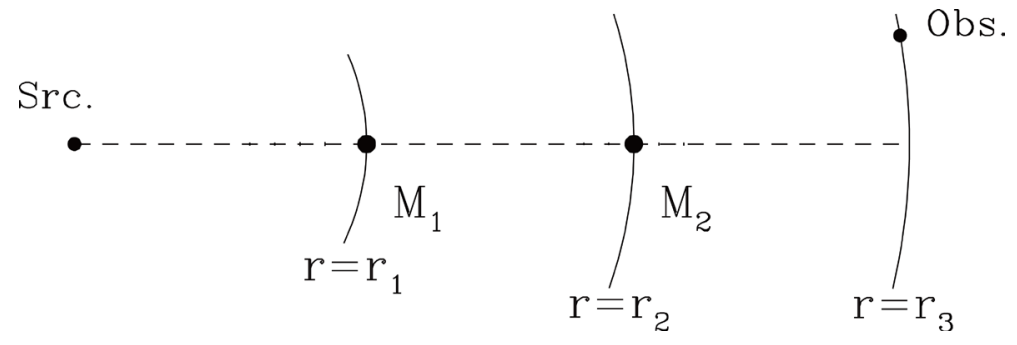

Figure 2. Lensing system considered in section 3.

explicit expression of Equation (6) is written as Equation (24) in Appendix (see also [6]). After some computation, the path integral expression reduces to (see Appendix for details)

$$
\begin{aligned}
F\left(r_{N}, \Theta_{N}\right)= & \frac{k}{2 \pi i} \frac{r_{1} r_{l_{2}}}{r_{l_{1}, l_{2}}} \int \mathrm{d}^{2} \Theta_{l_{1}} \exp \left[i k\left(\frac{r_{l_{1}} r_{l_{2}}}{2 r_{l_{1}, l_{2}}}\left|\Theta_{l_{1}}-\Theta_{l_{2}}\right|^{2}-\psi\left(\Theta_{l_{1}}\right)\right)\right] \\
& \times \frac{k}{2 \pi i} \frac{r_{l_{2}} r_{l_{3}}}{r_{l_{2}, l_{3}}} \int \mathrm{d}^{2} \Theta_{l_{2}} \exp \left[i k\left(\frac{r_{l_{2}} r_{l_{3}}}{2 r_{l_{2}, l_{3}}}\left|\Theta_{l_{2}}-\Theta_{l_{3}}\right|^{2}-\psi\left(\Theta_{l_{2}}\right)\right)\right] \\
& \times \cdots \\
& \times \frac{k}{2 \pi i} \frac{r_{l_{n}} r_{N}}{r_{l_{n}, N}} \int \mathrm{d}^{2} \Theta_{l_{n}} \exp \left[i k\left(\frac{r_{l_{n}} r_{N}}{2 r_{l_{n}, N}}\left|\Theta_{l_{n}}-\Theta_{N}\right|^{2}-\psi\left(\Theta_{l_{n}}\right)\right)\right]
\end{aligned}
$$

with

$$
\psi\left(\Theta_{l_{m}}\right)=2 \int_{r_{l_{m}}-\delta r}^{\eta_{l_{m}}+\delta r} \mathrm{~d} r U(r, \Theta)
$$

where $r_{l_{m-1}, l_{m}}=r_{l_{m}}-r_{l_{m-1}}, r_{l_{n+1}}=r_{N}$, and $\Theta_{l_{m}}$ is the variable on the $m$ th lens plane. As expected, stationary condition of the phase of the integrand in Equation (7) reproduces the lens equation in the multi-lens system [10]

$$
\nabla_{\Theta_{l_{m^{\prime}}}} \sum_{m=1}^{n}\left(\frac{r_{l_{m}} r_{l_{m+1}}}{2 r_{l_{m}, l_{m+1}}}\left|\Theta_{l_{m}}-\Theta_{l_{m+1}}\right|^{2}-\psi\left(\Theta_{l_{m}}\right)\right)=0,
$$

for each $m^{\prime}=1, \cdots, n$, which hints at a way to the geometrical optics limit. The Gaussian approximation around a stationary solution yields the result in the geometrical optics limit (see also [6]).

\section{Application to a Simple Configuration}

In this section we consider a simple case with two lenses. Figure 2 shows the 
configuration: The Schwarzschild lenses with mass $M_{1}$ and $M_{2}$ are located at the radius $r_{1}$ and $r_{2}$, respectively. The model considered here is not general, because the source and the two lenses are arranged to be on a straight line. However, this simplification allows us to perform integration of Equation (7) analytically. We start by rewriting Equation (7)

$$
\begin{aligned}
F= & \frac{k}{2 \pi i} \frac{r_{1} r_{2}}{r_{1,2}} \int \mathrm{d}^{2} \Theta_{1} \exp \left[i k\left(\frac{r_{1} r}{2 r_{1,2}}\left|\Theta_{1}-\Theta_{2}\right|^{2}-\psi\left(\Theta_{1}\right)\right)\right] \\
& \times \frac{k}{2 \pi i} \frac{r_{2} r_{3}}{r_{2,3}} \int \mathrm{d}^{2} \Theta_{2} \exp \left[i k\left(\frac{r_{2} r_{3}}{2 r_{2,3}}\left|\Theta_{2}-\Theta_{3}\right|^{2}-\psi\left(\Theta_{2}\right)\right)\right]
\end{aligned}
$$

with

$$
\psi\left(\Theta_{j}\right)=4 G M_{j} \ln \left(\left|\Theta_{j}\right|\right),
$$

for $j=1,2$, where $r_{i, j}=r_{j}-r_{i}$ and the position of an observer is $\left(r_{3}, \Theta_{3}\right)$. From Eqution (10) we have

$$
\begin{aligned}
F= & \frac{k}{i} \frac{r_{1} r_{2}}{r_{1,2}} \int_{0}^{\infty} \mathrm{d} \theta_{1} \theta_{1}^{1-4 i k G M_{1}} \exp \left[i k \frac{r_{1} r_{2}}{2 r_{1,2}}\left(\theta_{1}^{2}+\theta_{2}^{2}\right)\right] J_{0}\left(\frac{k r_{1} r_{2}}{r_{1,2}} \theta_{1} \theta_{2}\right) \\
& \times \frac{k}{i} \frac{r_{2} r_{3}}{r_{2,3}} \int_{0}^{\infty} \mathrm{d} \theta_{2} \theta_{2}^{1-4 i k G M_{2}} \exp \left[i k \frac{r_{2} r_{3}}{2 r_{2,3}}\left(\theta_{2}^{2}+\theta_{3}^{2}\right)\right] J_{0}\left(\frac{k r_{2} r_{3}}{r_{2,3}} \theta_{2} \theta_{3}\right),
\end{aligned}
$$

where $\left|\Theta_{j}\right|=\theta_{j}$ and $J_{0}(y)$ is the Bessel function of the first kind. Integration with respect to $\theta_{1}$ can be performed (see [10])

$$
\begin{aligned}
F= & \mathrm{e}^{i \alpha} \mathrm{e}^{\pi k G M_{1}} \Gamma\left(1-2 i k G M_{1}\right) \frac{k}{i} \frac{r_{2} r_{3}}{r_{2,3}} \int_{0}^{\infty} \mathrm{d} \theta_{2} \theta_{2}^{1-4 i k G M_{2}} \exp \left[i k\left(\frac{r_{1} r_{2}}{2 r_{1,2}}+\frac{r_{2} r_{3}}{2 r_{2,3}}\right) \theta_{2}^{2}\right] \\
& \times{ }_{1} F_{1}\left(1-2 i k G M_{1}, 1 ; \frac{-i k r_{1} r_{2}}{2 r_{1,2}} \theta_{2}^{2}\right) J_{0}\left(\frac{k r_{2} r_{3}}{r_{2,3}} \theta_{2} \theta_{3}\right),
\end{aligned}
$$

where $\alpha$ is a real number which represents a constant phase and ${ }_{1} F_{1}(a, b ; y)$ is the Kummer's function. With the use of the definition of the Bessel function

$$
J_{0}(z)=\sum_{L=0}^{\infty} \frac{(-1)^{L}}{(L !)^{2}}\left(\frac{z}{2}\right)^{2 L},
$$

we have (see [11])

$$
\begin{aligned}
F= & \mathrm{e}^{i \alpha^{\prime}} \mathrm{e}^{\pi k G\left(M_{1}+M_{2}\right)} \Gamma\left(1-2 i k G M_{1}\right) z \sum_{L=0}^{\infty} \frac{(-i)^{L}}{(L !)^{2}} \Gamma\left(1+L-2 i k G M_{2}\right) \\
& \times(x z)_{2}^{L} F_{1}\left(1-2 i k G M_{1}, 1+L-2 i k G M_{2}, 1 ; 1-z\right),
\end{aligned}
$$

where we defined

$$
\begin{aligned}
& z=\frac{r_{3}\left(r_{2}-r_{1}\right)}{r_{2}\left(r_{3}-r_{1}\right)}, \\
& x=\frac{k r_{2} r_{3} \theta_{3}^{2}}{2\left(r_{3}-r_{2}\right)},
\end{aligned}
$$


$\alpha^{\prime}$ is a real constant and ${ }_{2} F_{1}(a, b, c ; y)$ is the Hypergeometric function. In the limit $\theta_{3}=0(x=0)$, Eqution (15) reduces to

$$
\begin{aligned}
F= & \mathrm{e}^{i \alpha^{\prime}} \mathrm{e}^{\pi k G\left(M_{1}+M_{2}\right)} \Gamma\left(1-2 i k G M_{1}\right) \Gamma\left(1-2 i k G M_{2}\right) z \\
& \times{ }_{2} F_{1}\left(1-2 i k G M_{1}, 1-2 i k G M_{2}, 1 ; 1-z\right),
\end{aligned}
$$

and we have

$$
|F|^{2}=\left.\left.\frac{4 \pi k G M_{1}}{1-\mathrm{e}^{-4 \pi k G M_{1}}} \frac{4 \pi k G M_{2}}{1-\mathrm{e}^{-4 \pi k G M_{2}}} z^{2}\right|_{2} F_{1}\left(1-2 i k G M_{1}, 1-2 i k G M_{2}, 1 ; 1-z\right)\right|^{2} .
$$

We consider the coincidence limit that the distance between the two lenses becomes zero, i.e., $r_{1}=r_{2}$. In this case, from previous investigation (e.g., [10]), $F$ should be

$$
\mathcal{F}(x) \equiv \mathrm{e}^{\pi k G\left(M_{1}+M_{2}\right)} \Gamma\left(1-2 i k G M_{1}\right)_{1} F_{1}\left(1-2 i k G\left(M_{1}+M_{2}\right), 1 ;-i x\right),
$$

excepting a constant phase factor. We compare our result with Eqution (20). First, let us consider the limit $r_{1}=r_{2}$, i.e., $z=0$ of Eqution (15). Using the mathematical formula

$$
\begin{aligned}
\Gamma\left(1-2 i k G M_{1}\right) \Gamma\left(1-2 i k G M_{2}\right) z_{2} F_{1}\left(1-2 i k G M_{1}, 1-2 i k G M_{2}, 1 ; 1-z\right) \\
=z^{2 i k G\left(M_{1}+M_{2}\right)} \Gamma\left(1-2 i k G\left(M_{1}+M_{2}\right)\right) \\
\quad \times{ }_{2} F_{1}\left(2 i k G M_{1}, 2 i k G M_{2},-1+2 i k G\left(M_{1}+M_{2}\right) ; z\right) \\
\quad+z \frac{\Gamma\left(1-2 i k G M_{1}\right) \Gamma\left(1-2 i k G M_{2}\right) \Gamma\left(-1+2 i k G\left(M_{1}+M_{2}\right)\right)}{\Gamma\left(2 i k G M_{1}\right) \Gamma\left(2 i k G M_{2}\right)} \\
\quad \times{ }_{2} F_{1}\left(1-2 i k G M_{1}, 1-2 i k G M_{2}, 2-2 i k G\left(M_{1}+M_{2}\right) ; z\right),
\end{aligned}
$$

in the case $\theta_{3}=0(x=0)$, we can easily have

$$
\lim _{z \rightarrow 0}|F|^{2}=\frac{4 \pi k G\left(M_{1}+M_{2}\right)}{1-\mathrm{e}^{-4 \pi k G\left(M_{1}+M_{2}\right)}}=|\mathcal{F}(0)|^{2} .
$$

This is the expected result. Note that $|F|^{2}$ is regarded as the magnification factor. Figure 3 plots $R=|F / \mathcal{F}(0)|$, as a function of $Z$, where we fixed $k G M_{1}=k G M_{2}=1$. Thus the maximum magnification depends on lens configuration significantly. Figure 4 plots $R=|F / \mathcal{F}(x)|$ as a function of $x$ when fixing $z=0.5$ and $k G M_{1}=k G M_{2}=1$, which indicates that interference pattern also depends on lens configuration. In these figures we have fixed

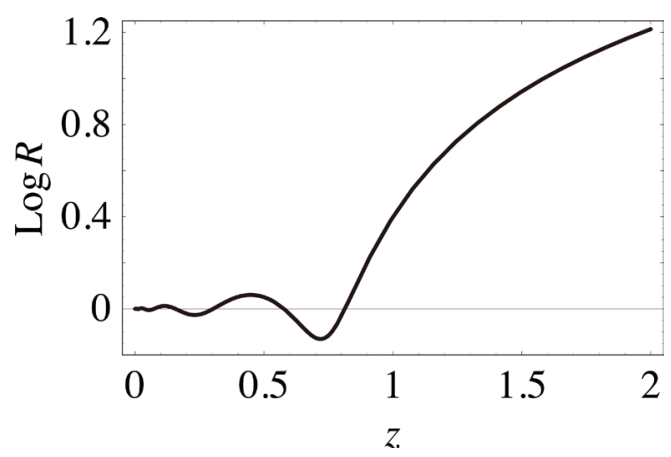

Figure 3. $R=|F / \mathcal{F}(0)|$ as a function of $z$ with fixing $k G M_{1}=k G M_{2}=1$. 


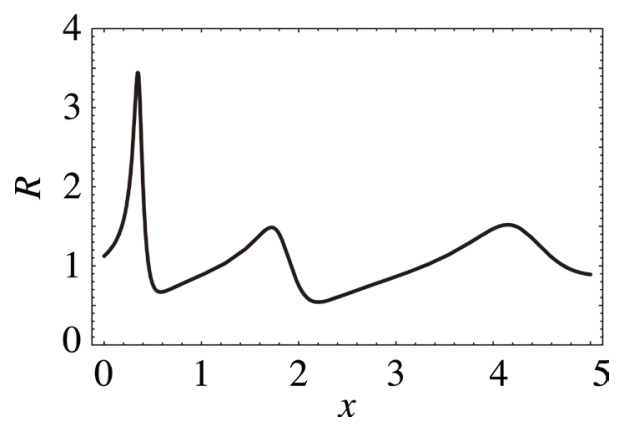

Figure 4. $R=|F / \mathcal{F}(x)|$ as a function of $x$ with fixing $z=0.5$ and $k G M_{1}=k G M_{2}=1$.

$k G M_{1}=k G M_{2}=1$, but result depends significantly on the parameters $k G M_{1}$, $k G M_{2}$ and $z$, as shown in Figure 4, which suggests variety of wave effect depending on lens configuration.

\section{Summary and Conclusion}

In the present paper, we have presented a general formula to investigate wave effect in multi-lens system, which has been derived with the use of path integral approach. The formula is expressed in terms of integration with respect to variables of lens planes. It is difficult to perform the integration in general cases, but a system with two Schwarzschild lenses is an example for which the integration can be performed in an analytic way. The model considered in the present paper is simplified and limited, however, it suggests variety of wave effect in multi-lens phenomenon. It is required to develop a numerical method to perform integration in general lens configuration in future work.

\section{Acknowledgements}

This work is supported in part by Grant-in-Aid for Scientific research of Japanese Ministry of Education, Culture, Sports, Science and Technology, No. 15740155.

\section{References}

[1] Takahashi, R. and Nakamura, T. (2003) Wave Effects in the Gravitational Lensing of Gravitational Waves from Chirping Binaries. The Astrophysical Journal, 595, 1039-1051. https://doi.org/10.1086/377430

[2] Seto, N. (2004) Strong Gravitational Lensing and Localization of Merging Massive Black Hole Binaries with LISA. Physical Review D, 69, Article ID: 022002 https://doi.org/10.1103/PhysRevD.69.022002

[3] Yamamoto, K. and Tsunoda, K. (2003) Wave Effect in Gravitational Lensing by a Cosmic String. Physical Review D, 68, Article ID: 041302. https://doi.org/10.1103/PhysRevD.68.041302

[4] De Paolis, F., Ingrosso, G., Nucita, A.A. and Qadir, A. (2002) A Note on Gravitational Wave Lensing. Astronomy and Astrophysics, 394, 749-752. https://doi.org/10.1051/0004-6361:20021258

[5] Baraldo, C., Hosoya, A. and Nakamura, T.T. (1999) Gravitationally Induced Inter- 
ference of Gravitational Waves by a Rotating Massive Object. Physical Review $D$, 59, Article ID: 083001. https://doi.org/10.1103/PhysRevD.59.083001

[6] Nakamura, T.T. and Deguchi, S. (1999) Wave Optics in Gravitational Lensing. Progress of Theoretical Physics Supplement, 133, 137-153. https://doi.org/10.1143/PTPS.133.137

[7] Nakamura, T.T. (1998) Gravitational Lensing of Gravitational Waves from Inspiraling Binaries by a Point Mass Lens. Physical Review Letters, 80, 1138-1141. https://doi.org/10.1103/PhysRevLett.80.1138

[8] Deguchi, S. and Watson, W.D. (1986) Wave Effects in Gravitational Lensing of Electromagnetic Radiation. Physical Review D (Particles and Fields), 34, 1708-1718. https://doi.org/10.1103/PhysRevD.34.1708

[9] Deguchi, S. and Watson, W.D. (1986) Diffraction in Gravitational Lensing for Compact Objects of Low Mass. Astrophysical Journal, Part 1, 307, 30-37. https://doi.org/10.1086/164389

[10] Schneider, P., Ehlers, J. and Falco, E.E. (1992) Gravitational Lenses. Springer-Verlag, Berlin.

[11] Magnus, W., Oberhettinger, F. and Soni, R.P. (1966) Formulas and Theorems for the Special Functions of Mathematical Physics. Springer-Verlag, Berlin.

https://doi.org/10.1007/978-3-662-11761-3 


\section{A. Derivation of Equation (7)}

In this Appendix, we review derivation of Equation (7) from Equation (6). We consider the configuration depicted as Figure 1. The source is located at the origin of coordinate and the position of an observer is specified by $(r, \Theta)=\left(r_{N}, \Theta_{N}\right)$. The space between the source and the observer is discritized by $N-1$ planes. The radius of each plane is labeled by $r_{j}$ for $j=1, \cdots, N-1$. Note that $r_{N}$ specifies the plane of the observer. $\Theta_{j}$ is (angle) variable on the th plane. We consider the system with $n$ lenses, and assume that $m$ th lens is located at the radius $r_{l_{m}}$. Assuming the validity of the thin lens approximation, we introduce the two dimensional potential

$$
\psi\left(\Theta_{l_{m}}\right)=2 \int_{r_{l}-\delta r}^{\eta_{+}+\delta r} \mathrm{~d} r U(r, \Theta),
$$

for $m=1, \cdots, n$, respectively. In this case, the path integral formula (6) can be written as

$$
F=\left[\prod_{j=1}^{N-1} \int \frac{\mathrm{d}^{2} \Theta_{j}}{A_{j}}\right] \exp \left[i k\left(\epsilon \sum_{j=1}^{N-1} \frac{r_{j} r_{j+1}}{2}\left|\frac{\Theta_{j+1}-\Theta_{j}}{\epsilon}\right|^{2}-\sum_{m=1}^{n} \psi\left(\Theta_{l_{m}}\right)\right),\right.
$$

where the normalization is chosen

$$
A_{j}=\frac{2 \pi i \epsilon}{k r_{j} r_{j+1}},
$$

so that $F=1$ in the limit $\psi=0$. Then, Equation (24) is rephrased as

$$
\begin{aligned}
F= & {\left[\prod_{j=1}^{l_{1}-1} \int \frac{\mathrm{d}^{2} \Theta_{j}}{A_{j}}\right] \exp \left[i k\left(\epsilon \sum_{j=1}^{l_{1}-1} \frac{r_{j} r_{j+1}}{2}\left|\frac{\Theta_{j+1}-\Theta_{j}}{\epsilon}\right|^{2}\right)\right] } \\
& \times\left[\prod_{j=l_{1}}^{l_{2}-1} \frac{\mathrm{d}^{2} \Theta_{j}}{A_{j}}\right] \exp \left[i k\left(\epsilon \sum_{j=l_{1}}^{l_{2}-1} \frac{r_{j} r_{j+1}}{2}\left|\frac{\Theta_{j+1}-\Theta_{j}}{\epsilon}\right|^{2}-\psi\left(\Theta_{l_{1}}\right)\right)\right] \\
& \times \cdots \\
& \times\left[\prod_{j=l_{n}}^{N-1} \frac{\mathrm{d}^{2} \Theta_{j}}{A_{j}}\right] \exp \left[i k\left(\epsilon \sum_{j=l_{n}}^{N-1} \frac{r_{j} r_{j+1}}{2}\left|\frac{\Theta_{j+1}-\Theta_{j}}{\epsilon}\right|^{2}-\psi\left(\Theta_{l_{n}}\right)\right),\right.
\end{aligned}
$$

where $\epsilon$ is the separation between two neighboring planes. With the use of the following equality, which can be proven by the mathematical induction,

$$
\sum_{j=l_{m}}^{l_{m+1}-1} r_{j} r_{j+1}\left|\Theta_{j+1}-\Theta_{j}\right|^{2}=\epsilon \frac{r_{l_{m}} r_{l_{m+1}}}{r_{l_{m+1}}-r_{l_{m}}}\left|\Theta_{l_{m+1}}-\Theta_{l_{m}}\right|^{2}+\sum_{j=l_{m}+1}^{l_{m+1}-1} r_{j}^{2} \frac{r_{j+1}-r_{l_{m}}}{r_{j}-r_{l_{m}}}\left|\Theta_{j}-u_{l_{m}, j}\right|^{2}
$$

with

$$
u_{l_{m}, j}=\frac{r_{l_{m}} \Theta_{l_{m}}+\left(j-l_{m}\right) r_{j+1} \Theta_{j+1}}{j\left(r_{j+1}-r_{l_{m}}\right)},
$$

we have 


$$
\begin{aligned}
F= & \int \mathrm{d}^{2} \Theta_{l_{1}} \frac{k}{2 \pi i} \frac{r_{l_{1}} r_{l_{2}}}{\left(r_{l_{2}}-r_{l_{1}}\right)} \exp \left[i k\left(\frac{r_{l_{1}} r_{l_{2}}}{2\left(r_{l_{2}}-r_{l_{1}}\right)}\left|\Theta_{l_{2}}-\Theta_{l_{1}}\right|^{2}-\psi\left(\Theta_{l_{1}}\right)\right]\right] \\
& \times \int \mathrm{d}^{2} \Theta_{l_{2}} \frac{k}{2 \pi i} \frac{r_{l_{2}} r_{l_{3}}}{\left(r_{l_{3}}-r_{l_{2}}\right)} \exp \left[i k\left(\frac{r_{l_{2}} r_{l_{3}}}{2\left(r_{l_{3}}-r_{l_{2}}\right)}\left|\Theta_{l_{3}}-\Theta_{l_{2}}\right|^{2}-\psi\left(\Theta_{l_{2}}\right)\right)\right] \\
& \times \cdots \\
& \times \int \mathrm{d}^{2} \Theta_{l_{n}} \frac{k}{2 \pi i} \frac{r_{l_{n}} r_{N}}{\left(r_{N}-r_{l_{n}}\right)} \exp \left[i k\left(\frac{r_{l_{n}} r_{N}}{2\left(r_{N}-r_{l_{n}}\right)}\left|\Theta_{N}-\Theta_{l_{n}}\right|^{2}-\psi\left(\Theta_{l_{n}}\right)\right)\right],
\end{aligned}
$$

which is equivalent to Eqution (7).

Submit or recommend next manuscript to SCIRP and we will provide best service for you:

Accepting pre-submission inquiries through Email, Facebook, LinkedIn, Twitter, etc. A wide selection of journals (inclusive of 9 subjects, more than 200 journals) Providing 24-hour high-quality service User-friendly online submission system Fair and swift peer-review system Efficient typesetting and proofreading procedure Display of the result of downloads and visits, as well as the number of cited articles Maximum dissemination of your research work

Submit your manuscript at: http://papersubmission.scirp.org/ Or contact ijaa@scirp.org 